\title{
Hypereosinophilic Cardiac Involvement Presenting With Left Ventricular Massive Thrombus and Cardioembolic Stroke: A Case Report
}

\author{
Muhammet Gurdogan ${ }^{1 *}$, Ugur Ozkan¹, Servet Altay, Fulya Puyan² \\ ${ }^{1}$ Department of Cardiology, Faculty of Medicine, Trakya University, Edirne, Turkey \\ ${ }^{2}$ Department of Pathology, Faculty of Medicine, Trakya University, Edirne, Turkey
} *Corresponding Author: Muhammet Gurdogan, M.D., Instructor, Department of Cardiology, Faculty of Medicine,
Trakya University, Edirne, Turkey. Tel: +90-2842351041, Fax: +90-2842351041, Email: drmgurdogan@gmail.com

Received March 10, 2018; Accepted June 19, 2018; Online Published August 29, 2018

\begin{abstract}
Introduction: It is well known that the tendency toward thrombosis is increased in cancer patients. The increase in cancer procoagulant and tissue factor levels, endothelial damage, and stasis due to compression are among the most accused causes of thrombosis in cancer patients. Hypereosinophilia is a rare condition that causes endothelial damage leading to thrombosis.

Case Presentation: We present a 64-year-old male patient with cardiac involvement of hypereosinophilia which developed in the T-cell lymphoma ground resulting in a fatal cardioembolic stroke. Despite normal left ventricular (LV) contractions, almost half of the ventricular volume was full of thrombus in this case.

Conclusion: Hypereosinophilia is a rare cause of thrombus formation in the left ventricle in patients with preserved ejection fraction. However, hypereosinophilic cardiac involvement can lead to rapid, progressive, life-threatening complications.

Keywords: Eosinophilia, Cardiac, Left Ventricular, Thrombosis, Stroke
\end{abstract}

\section{Introduction}

Thrombus formation in the ventricle of patients with normal left ventricular (LV) wall motion is a rare condition. A thrombus is usually expected to occur in the presence of an underlying facilitating cause, such as dilated cardiomyopathy, LV aneurysm, or myocardial infarction. ${ }^{1}$ Hypereosinophilia indicates that the number of eosinophils in the peripheral blood is greater than $1500 / \mu \mathrm{L}$. The number of eosinophils normally ranges from 3\%-5\% in the peripheral blood, and the absolute eosinophil count is $350-500 / \mu \mathrm{L} .{ }^{2}$ Eosinophilic infiltration and toxic mediators released from eosinophil granules can damage many organ systems. Although the skin, lungs, and gastrointestinal system are the organs most affected by hypereosinophilia, the most important cause of morbidity and mortality is heart involvement, which occurs in $20 \%$ of cases. ${ }^{2}$ The initial symptoms of hypereosinophilia are usually silent, which is why most cases are detected by chance. However, some cases present themselves with rapid, progressive, lifethreatening cardiac and neurological complications. ${ }^{3}$

The current study discusses a case of hypereosinophilia in which a massive thrombosis was seen in the LV despite the preservation of systolic functions in a patient with cardioembolic stroke etiology.

\section{Case Presentation}

A 64-year-old male patient was admitted to the emergency department with a sudden loss of weakness in his right leg. Physical examination showed TA: 110/70 mm Hg; heart rate, 105 beats/min; and saturation, 95\% (with pulse oximetry). No characteristics were present in the heart or lungs on auscultation. The abdomen was relaxed, but the Traube's space was closed. The spleen was palpable 2 $\mathrm{cm}$ below the left costa. After neurological evaluation, a cerebrovascular event was considered. Diffusion magnetic resonance imaging revealed early lacunar infarcts in both cerebral hemispheres (Figure 1A).

The history of the patient revealed that he had been admitted to the hematology department 1 month earlier because he had been diagnosed with hypereosinophilic syndrome based on T-cell lymphoma. He was discharged after 2 weeks with a daily, single subcutaneous dose of tinzaparin 14000 international units (IU)/0.7 mL vial and hydroxyurea treatment (Figure 1B). During

Copyright (C 2018 The Author(s). This is an open-access article distributed under the terms of the Creative Commons Attribution License (http:// creativecommons.org/licenses/by/4.0), which permits unrestricted use, distribution, and reproduction in any medium, provided the original work is properly cited. 


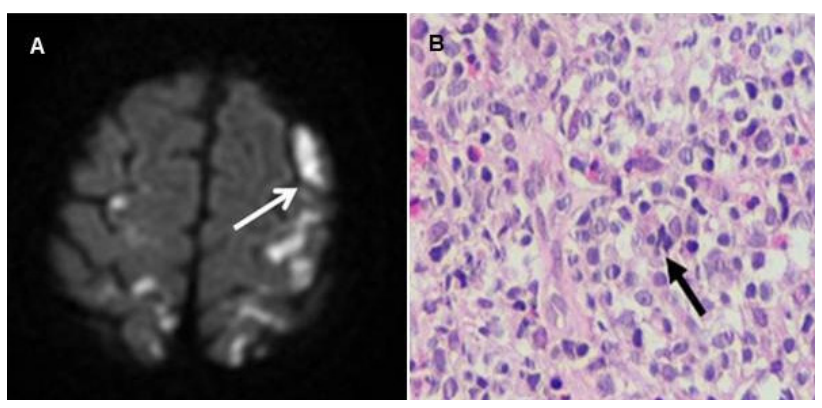

Figure 1. Cranial Magnetic Resonance Imaging: Early Lacunar Infarcts in Both Cerebral Hemispheres (A); A lymph node Trucut biopsy showed atypical heterogeneous lymphoid proliferation admixed with eosinophilic infiltration (B).

the patient's hospitalization period, a malignancy and a spleen thrombosis were detected, and the levels of protein $\mathrm{C}$, protein $\mathrm{S}$, homocysteine, lupus anticoagulant, anticardiolipin antibody, antinuclear antibody (ANA), and anti-neutrophil cytoplasmic antibody (ANCA), which can cause hypercoagulability, were checked, but the results were normal. An echocardiography examination during this hospitalization revealed normal LV systolic functions (ejection fraction [EF]: 57\%). No LV diastolic dysfunction (grade 1) was detected in the patient (Figure 2A).

The laboratory findings of the patient in the emergency department were as follows: leukocyte count, 11500/ $\mu \mathrm{L}$; eosinophil count, $2600 / \mu \mathrm{L}$ (percentage of eosinophils $22.5 \%$ ); and troponin level, $0.72 \mu \mathrm{g} / \mathrm{L}$ (normal range: 0.01-0.23 $\mu \mathrm{g} / \mathrm{L})$. Biochemical tests; platelet count; active partial thromboplastin time (APTT), prothrombin time (PT), and international normalized ratio (INR) values; and urine analysis were normal. The patient's electrocardiogram showed a normal sinus rhythm. No ischemic change was observed. Echocardiography was performed to investigate cardioembolic etiology due to stroke. The LV diameters and contractions of the patient were normal. Diastolic dysfunction (grade 1) was detected in the LV. The EF was measured as 55\% using the Simpson method. No valve pathology was reported. In the LV apex, an echogenic structure, which filled almost half the ventricular volume, was observed (Figure 2B). The patient's medical history, laboratory findings, the fact that he referred with a stroke, and echocardiographic findings suggested a hypereosinophilic cardiac involvement. A hematology consultation was requested, and the patient was hospitalized. The patient, who did not accept surgical treatment, was started on a regimen of enoxaparin 8000 anti-Xa IU/0.4 mL subcutaneously every 12 hours. The patient developed a cardiopulmonary arrest immediately after a second stroke the day after his hospitalization, did not respond to resuscitation, and died.

\section{Discussion}

Hypereosinophilia is a multisystemic disease with a poor prognosis. The most typical example of eosinophilic organ damage is cardiac involvement resulting in thrombus

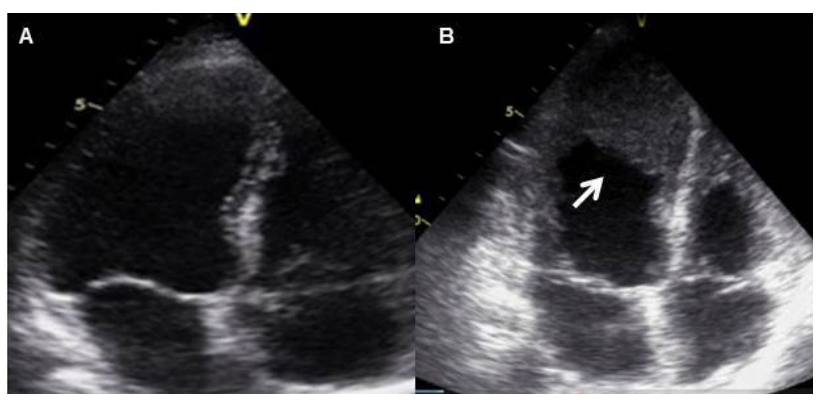

Figure 2. Left ventricular functions are normal, with no intracardiac mass image (A); Massive thrombus image was seen in the left ventricle (B).

formation, progressive heart failure, and restrictive cardiomyopathy. Cardiac involvement is the most important determinant of morbidity and mortality in hypereosinophilia. ${ }^{2,3}$ If not treated, approximately $75 \%$ of patients die within the first 3 years after diagnosis. ${ }^{4}$ It is known that heart failure due to hypereosinophilia occurs in three stages. The first is the acute necrotic period (in approximately 1.5 months); the second is the thrombotic period (in approximately 10 months); and the last is the fibrotic period (in approximately 24 months). ${ }^{5}$ Patients in the acute necrotic period are usually clinically silent. In this period, eosinophilic infiltration occurs in the endocardium and myocardium, followed by eosinophilic degranulation, secretion of toxic mediators, and necrosis in the endocardial and myocardial spaces. ${ }^{6}$ In the second stage, a thrombus is formed on the injured endocardial surface, often in the apical region of both ventricles, more rarely in subvalvular structures, in the LV outflow tract, and in the atrium. ${ }^{6}$ Severe sequelae can occur because of embolization of the thrombus in the heart into the lungs, abdominal organs, extremities, and especially the central nervous system. ${ }^{6}$ At the last stage of heart involvement, fibrosis is developed in the endocardium and myocardium. This results in a process ranging from diastolic dysfunction to restrictive cardiomyopathy ${ }^{66}$

The present study has three remarkable aspects: (1) After the first diagnosis of hypereosinophilia, a massive thrombus was formed in the LV in a much shorter period than stated in the literature. (2) Despite the fact that the patient had a history of using anticoagulants for 1 week after his discharge, the formation of a massive thrombus suggests that the progression may be very rapid in these patients. (3) Although the echocardiographic examination of the patient did not have a predisposing reason for a thrombus formation, the clinical picture was developed. The literature has revealed that echocardiography and magnetic resonance imaging are the most suitable diagnostic methods for the detection of intracardiac thrombus.?

The literature has reported many etiologic causes, as seen in the present study, of thrombus formation in the ventricles of patients with normal LV function. These include Takotsubo cardiomyopathy, pheochromocytoma, 
inflammatory diseases such as ulcerative colitis, infectious causes such as human immunodeficiency virus, protein C or protein $S$ that increase thrombosis susceptibility, lack of antithrombin 3 and myeloproliferative diseases, malignancies, and Behcet disease. ${ }^{8,9}$ Anticoagulant medications and thrombolytics are the most commonly used treatment methods in cases of thrombus detected in the LV. Surgical treatment may be a good option for patients with a high embolic risk and in cases where thrombolytic therapy is expected to increase the risk. ${ }^{10}$

\section{Conclusion}

In patients with normal LV function, hypereosinophilic cardiac involvement is an important predisposing factor for intracardiac thrombus development. When evaluating these patients, laboratory findings should be evaluated carefully. Another point that should be emphasized with this case presentation is the reality that not only a predisposition to hypercoagulability in patients with hematologic malignancy, but also hypereosinophilia can cause the development of thrombus.

\section{Authors' Contributions}

Concept: MG; Design: MG; Supervision: MG, UO, SA, FP; Materials: MG, UO, FP; Data collection: MG, UO, FP; Analysis and/or interpretation: MG, UO, SA; Literature search: MG; and Writing: MG.

\section{Conflict of Interest Disclosures}

The authors declare that they have no conflicts of interest.

\section{Ethical Approval}

Written informed consent was obtained from the relatives of the patient for the publication of the case report and the accompanying images.

\section{References}

1. Alzand BS, Ilhan M. Thrombus in a normal left ventricle. Neth Heart J. 2008;16(1):24-25. doi:10.1007/BF03086113.

2. Gotlib J. World Health Organization-defined eosinophilic disorders: 2014 update on diagnosis, risk stratification, and management. Am J Hematol 2014;89(3):325-337. doi: 10.1002/ajh.23664.

3. Ogbogu PU, Rosing DR, Horne MK 3rd. Cardiovascular manifestations of hypereosinophilic syndromes. Immunol Allergy Clin North Am. 2007;27(3):457-475. doi:10.1016/j. iac.2007.07.001.

4. Schooley RT, FlaumMA, GralnickHRA, et. al. A clinicopathologic correlation of the idiopathic hypereosinophilic syndrome. II. Clinical manifestations. Blood. 1981;58(5):1021-1026.

5. Weller PF, Bubley GJ. The idiopathic hypereosinophilic syndrome. Blood. 1994;83(10):2759-2779.

6. Roufosse FE, Goldman M, Cogan E. Hypereosinophilic syndromes. Orphanet J Rare Dis. 2007;2:37. doi: 10.1186/17501172-2-37.

7. Shah R, Ananthasubramaniam K. Evaluation of cardiac involvement in hypereosinophilic syndrome: complementary roles of transthoracic, transesophageal, and contrast echocardiography. Echocardiography. 2006;23(8):689-691. doi:10.1111/j.1540-8175.2006.00288.x.

8. Ebrahimifar P, Shahabi J. Right ventricular thrombosis as a manifestation of Behçet's syndrome. ARYA Atheroscler. 2017;13(2):91-94

9. Eren NK, Emren SV, Duygu H, et.al. Left ventricular thrombus formation in a patient with normal ejection fraction. Arch Turk Soc Cardiol. 2013;41:625-628. doi: 10.5543/tkda.2013.71598.

10. Lee $\mathrm{CH}$, Chen $\mathrm{CC}$, Chern MS. Thrombolytic therapy for acute left atrial thrombus formation in one patient with heart failure and atrial fibrillation. Circ J. 2007;71(4):604-607. doi:10.1253/ circj.71.604. 